\title{
Influence of rapid thermal annealing on a 30 stack InAs/GaAs quantum dot infrared photodetector
}

\author{
K. Stewart, ${ }^{\text {a) }}$ M. Buda, J. Wong-Leung, L. Fu, and C. Jagadish \\ Department of Electronic Materials Engineering, The Research School of Physical Sciences \\ and Engineering, The Australian National University, Canberra ACT 0200, Australia \\ A. Stiff-Roberts and P. Bhattacharya \\ Solid State Electronics Laboratory, Department of Electrical Engineering and Computer Science, \\ University of Michigan, Ann Arbor, Michigan 48109-2122
}

(Received 12 May 2003; accepted 28 July 2003)

\begin{abstract}
In this article the effect of rapid thermal annealing (RTA) on a 30 stacked InAs/GaAs, molecular beam epitaxially grown quantum dot infrared photodetector (QDIP) device is studied. Temperatures in the range of $600-800{ }^{\circ} \mathrm{C}$ for $60 \mathrm{~s}$, typical of atomic interdiffusion methods are used. After rapid thermal annealing the devices exhibited large dark currents and no photoresponse could be measured. Double crystal x-ray diffraction and cross sectional transmission electron microscopy studies indicate that this could be the result of strain relaxation. V-shaped dislocations which extended across many quantum dot (QD) layers formed in the RTA samples. Smaller defect centers were observed throughout the as-grown sample and are also likely a strain relaxation mechanism. This supports the idea that strained structures containing dislocations are more likely to relax via the formation of dislocations and/or the propagation of existing dislocations, instead of creating atomic interdiffusion during RTA. Photoluminescence (PL) studies also found that Si related complexes developed in the Si doped GaAs contact layers with RTA. The PL from these Si related complexes overlaps and dominates the PL from our QD ground state. (C) 2003 American Institute of Physics. [DOI: $10.1063 / 1.1609634]$
\end{abstract}

\section{INTRODUCTION}

Over the past few years quantum dot infrared photodetectors (QDIPs) have attracted much attention due to their potential for better device performance compared to quantum well infrared photodetectors (QWIPs). The zero-dimensional nature and three-dimensional carrier confinement of quantum dots (QDs) should, in theory, give higher detector responsivity, higher operating temperature, and the ability to detect normal incident radiation. ${ }^{1}$ Semiconductor QDIPs are routinely grown in the Stranski-Krastanow (SK) growth mode, in which the large lattice mismatch (7.2\% for InAs on GaAs) leads to self-organized, coherently strained island growth after a few monolayers of layer by layer growth. Typical quantum dot densities of $10^{10}-10^{11} \mathrm{dots} / \mathrm{cm}^{2}$ are achieved in the SK growth mode $^{2}$ and multiple layers of QDs are required to achieve sufficient photocurrent. Strain accumulation generated by such stacking is a large concern as it can lead to the formation of misfit dislocations, degrading device performance. The stability of these highly strained structures after rapid thermal annealing (RTA) is of particular importance for the application of atomic interdiffusion techniques to QDIP devices. Atomic interdiffusion techniques, involving high temperature rapid thermal annealing, have been used to successfully tune the detection wavelength of QWIPs ${ }^{3,4}$ which is critical to the development of two and multicolor infrared detectors needed for future high resolution systems. Most studies undertaken to date ${ }^{5-9}$ have investigated the influence

\footnotetext{
${ }^{a)}$ Electronic mail: kks109@rsphysse.anu.edu.au
}

of annealing on quantum dot superlattices (i.e., very small GaAs spacers such that strong strain coupling occurs between the QD layers). For QDIP devices, wide GaAs spacers are required to reduce the tunneling and thermally assisted tunneling dark current components. ${ }^{10}$ In this study, we investigate the effects of RTA on a 30 stack InAs/GaAs quantum dot structure with $50 \mathrm{~nm}$ GaAs spacers using device processing and characterization, cross sectional transmission electron microscopy (XTEM), photoluminescence (PL), and double crystal x-ray diffraction (DCXRD) measurements.

\section{EXPERIMENT}

The 30 stack InAs/GaAs quantum dot structure was grown on a (100) semi-insulating GaAs substrate using a solid source Varian Gen-II molecular beam epitaxy system. First, a $1 \mu \mathrm{m}$ thick, Si doped $\left(n=2 \times 10^{18} \mathrm{~cm}^{-3}\right) \mathrm{GaAs}$ bottom contact was grown followed by a $500 \mathrm{~nm}$ undoped GaAs buffer layer at a temperature of $620^{\circ} \mathrm{C}$. The temperature was ramped down to $500{ }^{\circ} \mathrm{C}$ and $2.2 \mathrm{ML}$ of InAs deposited followed by a $15 \mathrm{~s}$ growth interrupt to form the quantum dots. The quantum dots were directly doped with $\mathrm{Si}$ (1 $\times 10^{18} \mathrm{~cm}^{-3}$ ). The dot layers were separated by $50 \mathrm{~nm}$ of undoped GaAs. Finally, the substrate temperature was ramped back to $620^{\circ} \mathrm{C}$ for the growth of a $1 \mu \mathrm{m}$ thick, $\mathrm{Si}$ doped $\left(n=2 \times 10^{18} \mathrm{~cm}^{-3}\right) \mathrm{GaAs}$ top contact layer. Figure 1 is an illustration of the device structure. Pieces were cleaved from the as-grown sample and RTA was performed on the separate pieces at 600,700 , or $800^{\circ} \mathrm{C}$ for $60 \mathrm{~s}$ under argon flow. During annealing the pieces were sandwiched between 


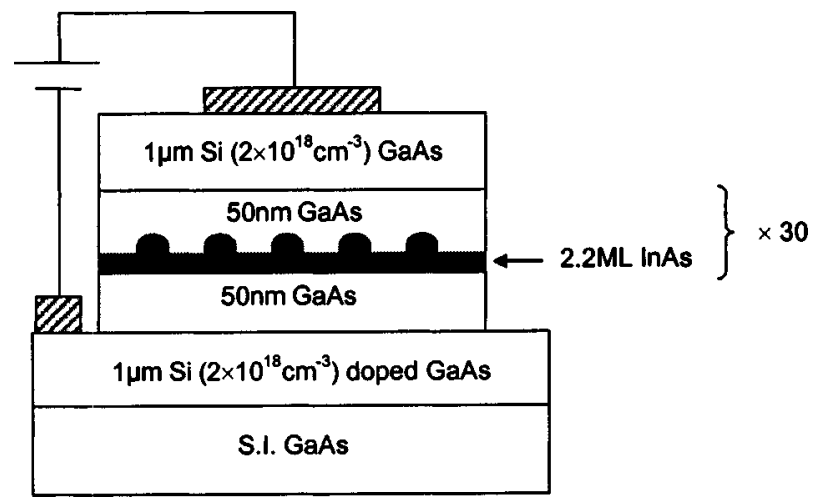

FIG. 1. Schematic of the quantum dot infrared photodetector.

two GaAs semi-insulating wafers (proximity capping) to prevent outdiffusion of arsenic. Devices $(250 \times 250 \mu \mathrm{m}$ mesas $)$ were fabricated from the as-grown and annealed samples using standard photolithography and wet etching, and Ge$\mathrm{Ni}-\mathrm{Au}$ ohmic contacts were evaporated onto the mesas. The detectors were connected to a leadless chip package and mounted in a liquid nitrogen cooled dewar for photocurrent and $I-V$ measurements. To gain a greater understanding of the device behavior, PL, XTEM, and DCXRD studies were performed. The XTEM study was performed using a Philips CM 300 electron microscope operated at $200 \mathrm{keV}$. Samples were glued face to face with epoxy and mechanically thinned to a thickness of $\sim 15 \mu \mathrm{m}$ followed by Ar ion milling at $3 \mathrm{kV}$ on a Gatan Duo mill at liquid nitrogen temperature. The DCXRD scans were measured with a Bede Scientific QC2a diffractometer using the $\mathrm{Cu} K \alpha_{1}$ radiation. All PL measurements were made at $77 \mathrm{~K}$ using a $533 \mathrm{~nm}$ diode pumped solid state laser.

\section{RESULTS}

\section{A. Photoluminescence}

Figure 2(a) shows typical PL spectra for the as-grown and annealed samples. Two emission peaks at $\sim 985$ and $\sim 1050 \mathrm{~nm}$ are present in the as-grown spectrum. These are labeled A and B in Fig. 2(a), respectively. The emission at $\sim 1050 \mathrm{~nm}(1.18 \mathrm{eV})$ is broad and may be due to $V_{\mathrm{Ga}}-\mathrm{Si}_{\mathrm{Ga}}$ defects within the heavily $\mathrm{Si}$ doped capping layer. ${ }^{11}$ The emission at $\sim 985 \mathrm{~nm}(1.26 \mathrm{eV}$ ) is narrower (FWHM $=50 \mathrm{~nm}$ ) and is attributed to the quantum dot ground state in agreement with previous studies of similar device structures. $^{12}$

After RTA at $700{ }^{\circ} \mathrm{C}\left(600^{\circ} \mathrm{C}\right)$ a new emission peak, labeled C in Fig. 2(a), is observed at $1190 \mathrm{~nm}(1215 \mathrm{~nm})$ with a large FWHM of $165 \mathrm{~nm}$. RTA also increases the intensity of peak A and shifts it to longer wavelength. For example, after RTA at $700{ }^{\circ} \mathrm{C}$ for $60 \mathrm{~s}$, peak A's intensity has tripled, its FWHM doubled and its wavelength redshifted by $10 \mathrm{~nm}$. This is not the typical behavior reported by other groups, ${ }^{5,6,8,9,13}$ where RTA of QD stacks leads to a blueshift in the PL spectrum and a narrowing of the FWHM due to interdiffusion of $\mathrm{Ga} / \mathrm{In}$ atoms at the QD interface. Initially strain relaxation was thought responsible for the observed redshift $^{14}$ (see TEM results discussed later). However, PL

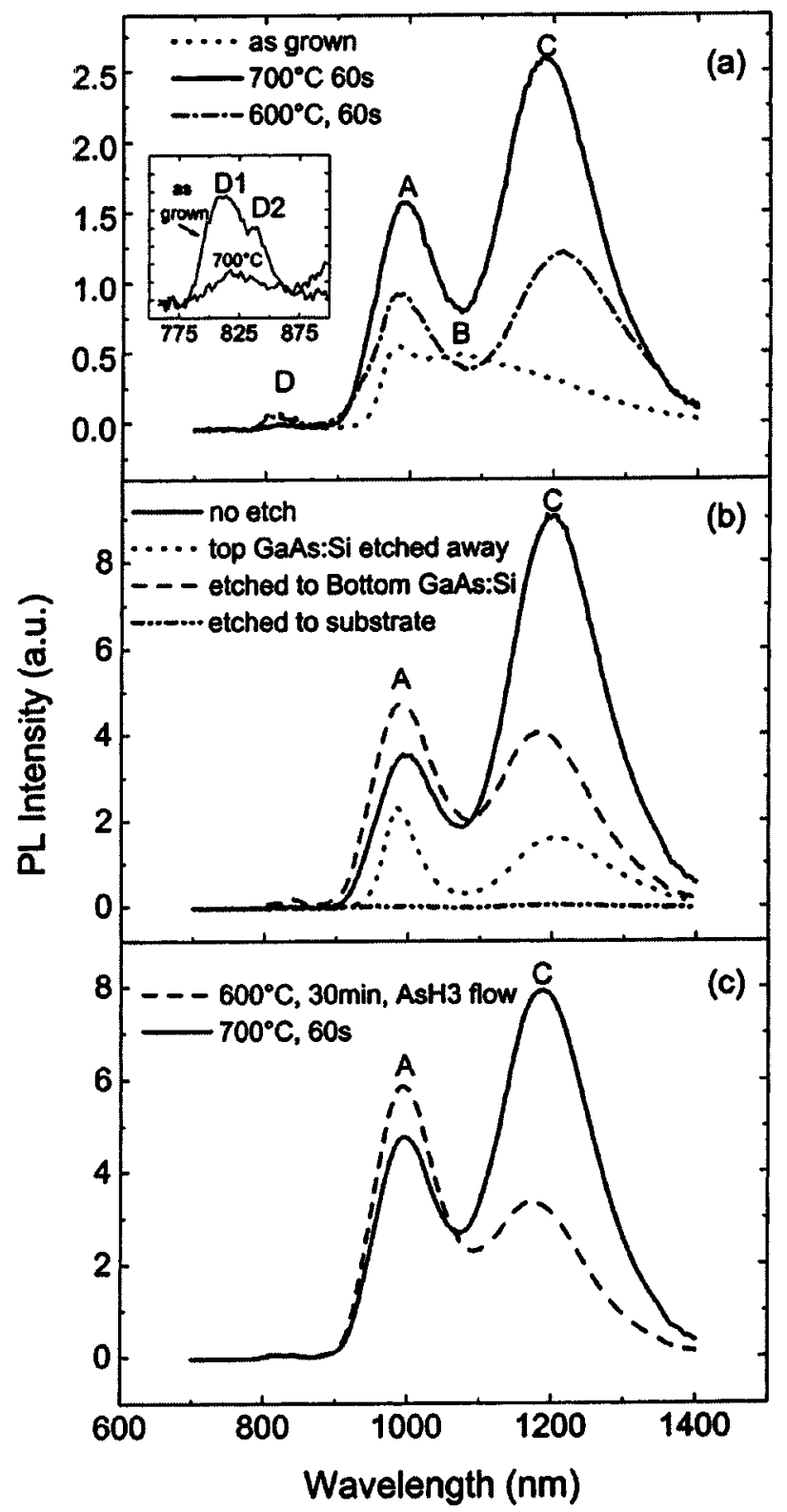

FIG. 2. Photoluminescence spectra at $77 \mathrm{~K}$ of (a) the as-grown QDIP sample and samples annealed at either 600 or $700{ }^{\circ} \mathrm{C}$ for $60 \mathrm{~s}$, (b) the QDIP sample annealed at $700{ }^{\circ} \mathrm{C}$ for $60 \mathrm{~s}$ and then etched to different depths, (c) the QDIP sample annealed for $30 \mathrm{~min}$ at $600{ }^{\circ} \mathrm{C}$ under arsine (the sample annealed at $700{ }^{\circ} \mathrm{C}$ for $60 \mathrm{~s}$ is shown for comparison): The inset in (a) shows more clearly the low intensity emission at $800 \mathrm{~nm}$ (labeled D).

studies after etching away the Si doped GaAs contact layer revealed that the redshift and increased intensity are associated with a defect in the thick GaAs contact layer, which has a strong luminescence overlapping with that of the quantum dots. As can be seen in Fig. 1, the device structure consists of 30 QD layers sandwiched between $1 \mu \mathrm{m}$ thick, Si doped $\left(2 \times 10^{18} \mathrm{~cm}^{-3}\right)$ top and bottom GaAs layers. It is within these heavily doped layers that we believe Si related defects are created. This is demonstrated with reference to Fig. 2(b), which shows PL spectra for a QDIP sample RTA at $700{ }^{\circ} \mathrm{C}$ for $60 \mathrm{~s}$ and then etched to different depths. After etching the top $1 \mu \mathrm{m}$ Si doped layer, the intensity of peaks $\mathrm{A}$ and $\mathrm{C}$ decreases significantly. Peak A is also blueshifted to $985 \mathrm{~nm}$ and its FWHM reduced by half. These characteristics are 
nearly identical to those of the original, as grown QDs and this PL is thought to come from the RTA QDs which are no longer in competition with the efficient $\mathrm{Si}$ related defect complexes. This also seems to indicate that there is negligible wavelength shift of the QD emission with RTA at $700{ }^{\circ} \mathrm{C}$, which is still contrary to the blueshift observed by other groups. ${ }^{5,6,8,9,13}$ With further etching, so that the QD layers are removed but the bottom GaAs:Si layer is still present, the PL and FWHM of peaks A and C increase again. It therefore appears that RTA leads to the formation of $\mathrm{Si}$ related complexes with strong PL at $995 \mathrm{~nm}$ (peak A) and $1200 \mathrm{~nm}$ (peak C). The strong emission at $995 \mathrm{~nm}$ dominates the ground state emission from the RTA QDs. This means that the increase in intensity of peak A with RTA is not due to an improved QD material quality.

Other investigators have reported a peak at $1.22 \mathrm{eV}$ $(1020 \mathrm{~nm})$ for Si doped GaAs that increases in intensity and shifts to higher energies in the $1.25 \mathrm{eV}(990 \mathrm{~nm})$ range after annealing under arsine, due to changes in the concentration of point defects. ${ }^{11,15,16}$ This emission band is attributed to the internal transition of electrons between the excited and ground states of a $\mathrm{Si}_{\mathrm{Ga}}-V_{\mathrm{Ga}}$ complex. Si clustering in conjunction with $\mathrm{Si}_{\mathrm{Ga}}-V_{\mathrm{Ga}}$ has also been suggested. ${ }^{17}$ This complex may be responsible for the $1050 \mathrm{~nm}$ emission (peak B) in our as-grown sample which shifts to $995 \mathrm{~nm}$ (peak A) with RTA.

A 30 min anneal at $600{ }^{\circ} \mathrm{C}$ under arsine flow was also performed. The PL spectrum of this sample is displayed in Fig. 2(c). The two Si-related defect peaks are still present, however compared to the $700{ }^{\circ} \mathrm{C}, 60 \mathrm{~s}$ RTA the intensity of the $1200 \mathrm{~nm}$ peak (peak C) is reduced. A similar effect is seen in Fig. 2(b), where peak C's intensity is lower in the PL spectrum from the bottom GaAs:Si layer than it is from the top layer. We believe this is related to the annealing conditions. Ga rich conditions tend to prevail during rapid thermal annealing using a proximity cap but would not influence the bottom GaAs layer as strongly. Under Ga rich conditions three native point defects are favorable: vacancies in the arsenic sublattice $\left(V_{\mathrm{As}}\right)$, gallium self interstitial $\left(I_{\mathrm{Ga}}\right)$, and a gallium atom sitting on an arsenic site $\left(\mathrm{Ga}_{\mathrm{As}}\right)$. We infer that the $1200 \mathrm{~nm}$ luminescence of peak $\mathrm{C}$ is related to a point defect complex which is associated with $\mathrm{Ga}$ rich conditions, involving $V_{\mathrm{As}}, I_{\mathrm{Ga}}$, and/or $\mathrm{Ga}_{\mathrm{As}}$ point defects. A number of articles have reported $1200 \mathrm{~nm}$ photoluminescence at $77 \mathrm{~K}$ in Si doped $\mathrm{GaAs}^{18,19}$ and correlate it with the $\mathrm{Si}_{\mathrm{Ga}}-\mathrm{Si}_{\mathrm{As}}$ complex. Although Liang et al. ${ }^{20}$ based on energetic considerations, also suggests $V_{\mathrm{Ga}}-V_{\mathrm{As}}$ pairs. Annealing under $\mathrm{Ga}$ rich conditions must therefore lead to an increased $V_{\mathrm{As}}$ and/or $\mathrm{Si}_{\mathrm{As}}$ concentration. Some likely reactions involving $V_{\mathrm{As}}, I_{\mathrm{Ga}}$, and $\mathrm{Ga}_{\mathrm{As}}$ point defects are listed below. As already mentioned $V_{\text {As }}$ point defects are favored under Ga rich conditions. Reaction (1) is a possible mechanism by which they may form. $I_{\mathrm{As}}$ formed during this reaction has a fast diffusion rate and migrates to the surface leaving an increased number of $V_{\mathrm{As}}$ behind. As can be seen from reaction (2), the concentration of $\mathrm{Si}_{\mathrm{As}}$ is linked to that of the $V_{\mathrm{As}}$ :

$$
\begin{aligned}
& \mathrm{As}_{\mathrm{As}} \leftrightarrow V_{\mathrm{As}}+I_{\mathrm{As}}, \\
& \mathrm{Si}_{\mathrm{Ga}}+V_{\mathrm{As}} \leftrightarrow V_{\mathrm{Ga}}+\mathrm{Si}_{\mathrm{As}},
\end{aligned}
$$
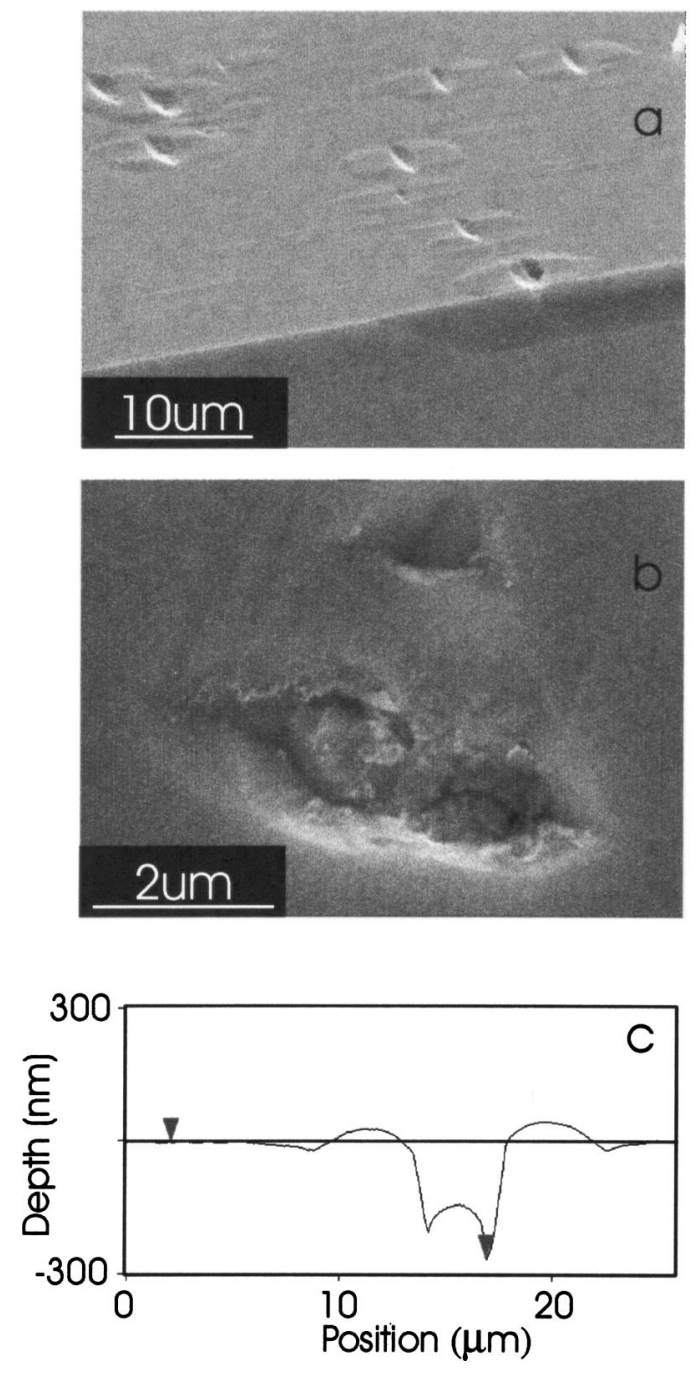

FIG. 3. Scanning electron micrographs of (a) a group of oval defects and (b) a single oval defect; (c) a line scan through an oval defect's long axis measured using contact mode atomic force microscopy.

$$
\mathrm{Ga}_{\mathrm{As}}+\mathrm{Si}_{\mathrm{Ga}} \leftrightarrow \mathrm{Si}_{\mathrm{As}}+\mathrm{Ga}_{\mathrm{Ga}} .
$$

The inset of Fig. 2(a) is an enlarged image of the low intensity emission (labeled D) at $800-850 \mathrm{~nm}$. On close examination this consists of two peaks. We attribute the first peak, D1, to exciton transitions in the undoped GaAs spacers and the second peak, D2, to either band-band transitions in the highly $\mathrm{Si}$ doped GaAs top contact or $\mathrm{Si}_{\mathrm{D}}-\mathrm{Si}_{\mathrm{A}}$ pairs. The GaAs emission is low compared to the defect peak B suggesting efficient defect related radiative recombination. After annealing these peaks decrease in intensity, possibly due to very efficient trapping of the photoexcited carriers by the $\mathrm{Si}$ related complexes.

Oval defects developed and increased in concentration with time for the as-grown QDIP structure and may also be adversely affecting the GaAs PL intensity. After growth the surface was mirror like with no oval defects. On inspection two months later a high density $\left(\sim 4200 / \mathrm{cm}^{2}\right)$ of oval defects had developed. Figures 3(a) and 3(b) show scanning electron micrographs (SEM) of these defects. The paired oval defects are aligned with their long axis parallel with one of the cleave planes. A pit cuts through the center perpendicular to 

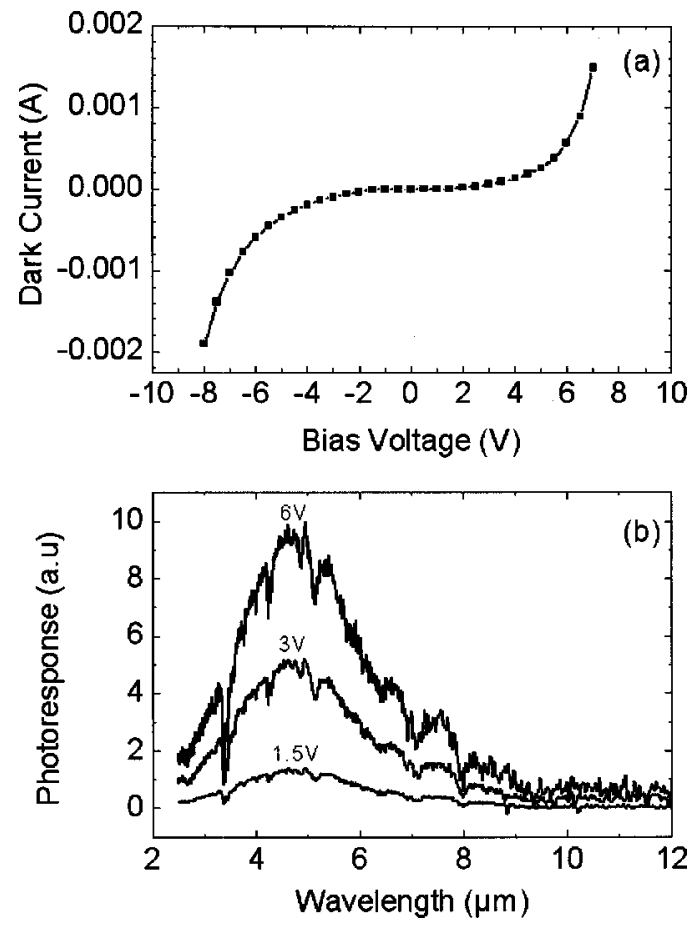

FIG. 4. Device characteristics measured for the QDIP at $77 \mathrm{~K}$; (a) dark current and (b) photoresponse for several applied biases.

the long axis. Also seen in the SEM image are thin raised regions, also parallel to the cleave plane, which may be an earlier stage of oval defect formation. As can be seen in Fig. 3(a), the oval defects tend to appear in groups, with regions in between which are relatively free of oval defects. Figure 3 (c) is a line scan through the long axis of a larger oval defect measured by atomic force microscopy (AFM). Along this axis the oval defects can be up to $14 \mu \mathrm{m}$ long and 260 $\mathrm{nm}$ in depth. It is not yet clear what is responsible for the generation of these oval defects. Possibilities include an insufficient bake out of the growth chamber, incomplete degassing of the $\mathrm{Ga}$ source or the formation of $\mathrm{Ga}$ droplets at the mouth of the Ga cell and their subsequent sputtering onto the substrate. Mehta et al. ${ }^{21}$ suggest that oval defects are formed as a result of microdroplets of liquid $\mathrm{Ga}$ which deposit onto the surface. If all of the $\mathrm{Ga}$ is unable to react with arsenic, the unreacted Ga remains enclosed in the GaAs crust. This liquid Ga bubble can become unstable and burst due to the high liquid Ga surface tension leading to oval defects. This may explain the development of the oval defects with time.

\section{B. Device Performance}

The $I-V$ and photocurrent characteristics for the asgrown device are displayed in Fig. 4. The peak detection wavelength is at $4.8 \mu \mathrm{m}$ with a FWHM of $2.6 \mu \mathrm{m}$, and $\Delta \lambda / \lambda=54 \%$ at a bias voltage of $-6 \mathrm{~V}$. There was no change in the peak detection wavelength with applied bias voltage. The large linewidth is typical of SK self-assembled QDs due to the random nucleation process and wide quantum dot size distribution. A similarly wide ( $F W H M=50 \mathrm{~nm})$ PL signal is observed for the as-grown sample [Fig. 2(a)]. It is also an indication that the transition is bound to continuum as ex-
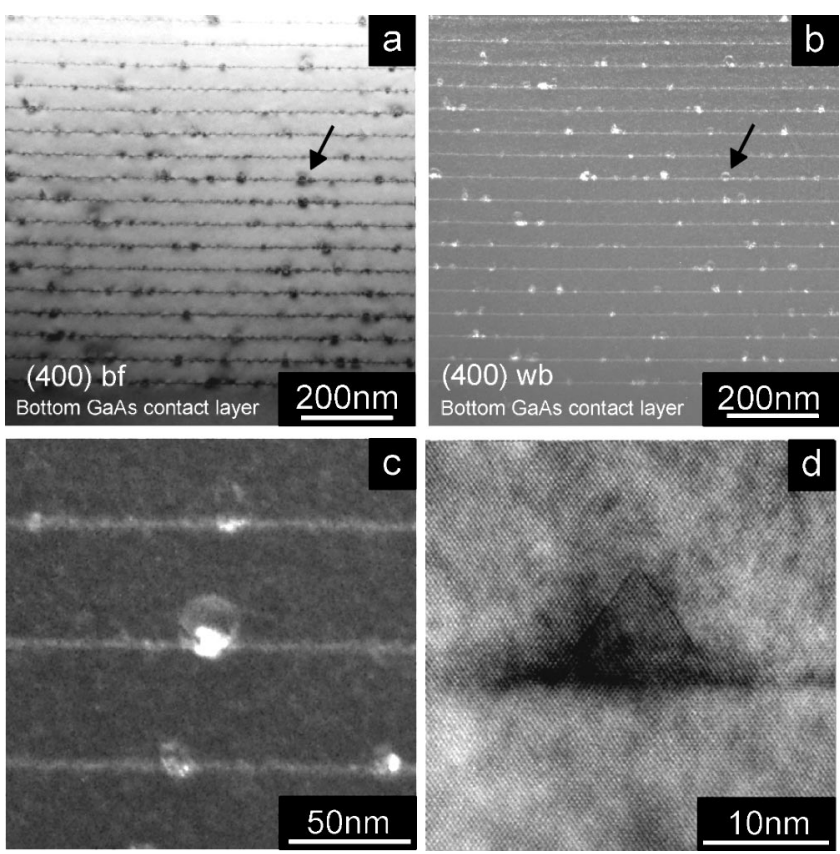

FIG. 5. Cross section transmission electron micrographs of the as-grown sample under the following imaging conditions: (a) (400) bright field, (b) (400) weak beam, (c) (400) weak beam, and (d) [011] zone axis: (c) is an enlarged image of a dislocation loop and (d) a high resolution image of an inverted $\mathrm{v}$-shaped dislocation.

pected from studies on similar device structures. ${ }^{2}$ Devices fabricated out of the RTA samples had large dark currents. At a bias voltage of only $0.5 \mathrm{~V}$ they had a dark current 4 orders of magnitude greater than those fabricated from the as-grown material. This large dark current obscured the photocurrent signal and prevented further measurement of device performance. The large dark current could possibly be a result of current leakage associated with the presence of dislocations and/or the Si related complexes. In order to gain a better understanding of the mechanisms by which RTA led to this high dark current, DCXRD and XTEM studies were also performed.

\section{Cross-sectional transmission electron microscopy}

Figures 5(a) and 5(b) show representative XTEM images of the as-grown sample. The bright field image in Fig. 5(a) shows a strong black and white contrast in the QD layers indicative of high strain close to the quantum dot layer. Strain in these regions is present around the WL, QDs, and defects. Figure 5(b) is a weak beam (WB) image of the same area and differentiates well between the different strain regions. The QDs show very little contrast, while the WL and defects (see solid black arrows) show strong contrast and are clearly revealed. Figures 5(c) and 5(d) are enlarged images of the two main defect types which are present in large numbers throughout the as-grown and the annealed samples. Figure 5(c) is a misfit dislocation loop that appears to be growing outwards from the QD layer. We also observed inverted V-shaped defects extending from the QD layer into the above GaAs spacer [Fig. 5(d)]. These typically have a $55^{\circ}$ incline to the (100) plane, indicating that they are gliding on the adjacent $\{111\}$ slip planes viewed edge on in this geometry. These 


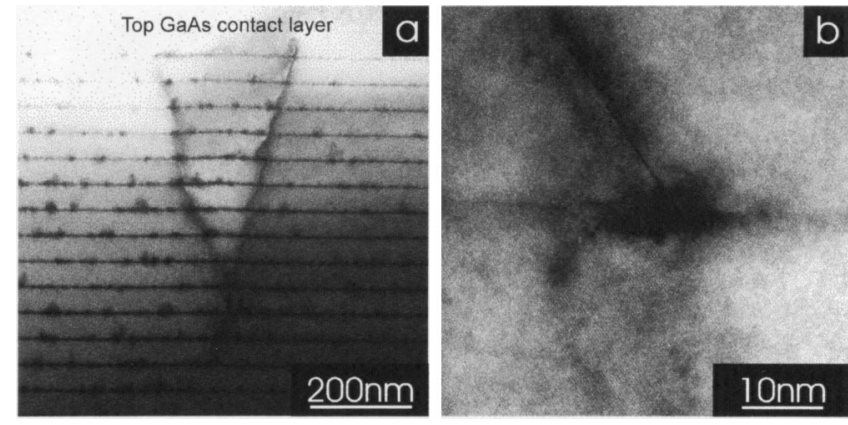

FIG. 6. Zone axis [011] transmission electron micrographs of the QDIP sample after RTA at $700{ }^{\circ} \mathrm{C}$ for $60 \mathrm{~s}$ showing (a) a large V-shaped dislocation and (b) one section of a large V-shaped dislocation where it crossed an InAs/GaAs interface.

defects may be an edge-on view of the loops described earlier as this geometry allows us to view only two $\{111\}$ planes edge on. Analyses of these defects show that the two sides of the inverted $\mathrm{v}$-shaped defects are actually two separate defects as they are invisible under different imaging $\mathbf{g}$ conditions. Further work is still needed to properly characterize these defects. We speculate that the growth of the dislocation loop outwards from the QD layer is a strain relief mechanism and may explain the low QD PL intensity relative to that from the Si-related complexes.

Of special interest are the extensive threading dislocations that form after rapid thermal annealing [Fig. 6(a)]. These originate at the layer interface and travel upwards through the GaAs spacers from one interface to the next. We note that these threading dislocations do not seem to show any preferential gliding on the $\{111\}$ planes clearly evident from the angle $(\theta)$ this defect makes with the QD layers $(\theta$ $\left.\neq 55^{\circ}\right)$. Again the two sides of the extensive V-shaped defects are two individual defects as confirmed by TEM analyses. Figure $6(\mathrm{~b})$ shows a high resolution image of one such dislocation originating at the InAs/GaAs interface. Shiramine et al. observed similar extensive threading dislocations. ${ }^{22,23}$ However these threading dislocations are created during growth and unlike our defects are clearly aligned at $55^{\circ}$ to the (001) plane and therefore glide on the $\{111\}$ planes. They propose that the dislocations form as a result of strain in the vicinity of two islands that nucleate close together during growth. Further work is in progress to better understand these defects and their origins.

In some regions, up to four of these extensive $\mathrm{V}$ defects were observed in a $1 \mu \mathrm{m}$ long section and may explain the failure of devices fabricated from this material. The presence of smaller defect centers within the as-grown sample and the formation of the larger dislocations after RTA may also explain why the QD ground state PL did not blueshift with RTA. Other groups ${ }^{13}$ have proposed that for structures in which some strain relaxation has already occurred via the formation of misfit dislocations, strain induced interdiffusion becomes negligible and instead RTA will tend to lead to the generation of further dislocations or propagation of existing dislocations.

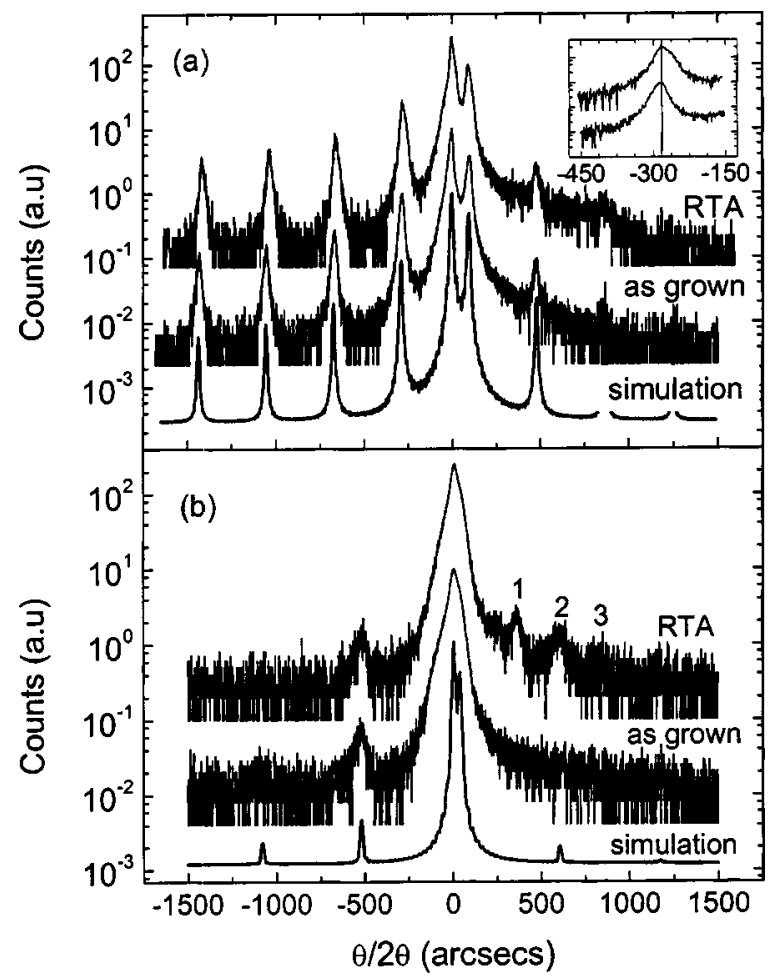

FIG. 7. X-ray rocking curves using (a) the (004) symmetric and (b) the (115) asymmetric reflection, for the as-grown QDIP sample and a QDIP sample repetitively annealed at 700,750 , and then $800^{\circ} \mathrm{C}$ for $60 \mathrm{~s}$. The spectra are shifted vertically for clarity: The inset in (a) shows the zeroth order peaks. Simulated curves are also shown.

On close inspection of Fig. 6(a), the material inside the extensive threading dislocation exhibits a different contrast to that outside. Also the wetting layers are slightly bent towards the top surface with a radius of curvature of 3-5 $\mu \mathrm{m}$. The radius of curvature of a two layer composite square plate due to lattice mismatch can be calculated using ${ }^{24}$

$$
\frac{1}{R}=\frac{12 \times \frac{K_{2}}{K_{1}} \frac{t_{2}}{t_{1}}}{t_{1}\left(1+6 \times \frac{K_{2}}{K_{1}} \frac{t_{2}}{t_{1}}\right)} \Delta \varepsilon,
$$

where $t$ is the layer thickness; $K=E /(1-\nu)$ for the biaxial stress case; $E$ is Young's modulus; $\nu$ is the Poisson's ratio, and $\Delta \varepsilon$ is the lattice mismatch which is $7.2 \%$ for InAs on GaAs. Subscripts 1 and 2 refer to the GaAs and InAs layers, respectively. Equation (4) gives a radius of curvature of 4 $\mu \mathrm{m}$ which agrees well with that determined from the XTEM image. Using this radius of curvature the GaAs tensile and InAs compressive strain parallel to the interface, $\epsilon_{\|}$can be calculated. ${ }^{24}$ A small amount of tensile strain $\left(\epsilon_{\|}=-0.007\right)$ was calculated for the GaAs. This strain calculation is later compared with the strain determined from x-ray diffraction.

\section{Double crystal x-ray diffraction}

Figures 7(a) and 7(b) show symmetric (004) and asymmetric, glancing incidence, (115) rocking curves from DCXRD measurements, respectively. Rocking curves are shown for the as-grown sample and a sample which was 
repetitively annealed at 700,750 , and $800{ }^{\circ} \mathrm{C}$ for $60 \mathrm{~s}$. The symmetric scan only gives us information about $a_{\perp}$, the lattice parameter parallel to the growth direction while the asymmetric scan is sensitive to both $a_{\perp}$ and the in-plane lattice constant, $a_{\|}$. Figures 7(a) and 7(b) also contain a simulated curve of the as-grown scans based on the TakagiTaupin equations of dynamical theory. It assumes a thin InAs/GaAs quantum well structure consisting of $7.7 \AA$ of InAs and $485 \mathrm{~nm}$ of GaAs. After repetitive RTA, the peaks in the symmetric pattern appear skewed to the right due to the formation of a second set of peaks [see Fig. 7(a)]. Compared to the first set of peaks, this second set is shifted towards the GaAs substrate indicating the onset of strain relaxation. Three tensile peaks, labeled 1, 2, and 3 [Fig. 7(b)], are also present in the (115) asymmetric rocking curve of the RTA sample but not in the as-grown sample. Peak 2 is predicted in the simulated curve however peaks 1 and 3 are definitely new and a result of RTA. It is possible that the additional peaks, seen in the symmetric and asymmetric XRD patterns, are due to the strain modified material within the large V-shaped dislocations. As calculated earlier, a slight amount of tensile strain may be accommodated by the GaAs within these defects.

\section{Strain estimates from XRD rocking curves}

The average perpendicular and parallel strain, related to the position of the zero order peak of the superlattice, can be estimated from:

$$
\begin{gathered}
\varepsilon_{\perp \text { average }}=\frac{\varepsilon_{\perp \mathrm{GaAs}} \times d_{\mathrm{GaAs}}+\varepsilon_{\perp \mathrm{InAs}} \times d_{\mathrm{InAs}}}{d_{\mathrm{InAs}}+d_{\mathrm{GaAs}}} \\
\varepsilon_{\| \text {average }}=\frac{\varepsilon_{\| \mathrm{GaAs}} \times d_{\mathrm{GaAs}}+\varepsilon_{\| \mathrm{InAs}} \times d_{\mathrm{InAs}}}{d_{\mathrm{InAs}}+d_{\mathrm{GaAs}}},
\end{gathered}
$$

where $d_{\mathrm{GaAs}}$ and $d_{\mathrm{InAs}}$ are the thickness of the GaAs and InAs layers, respectively, and

$$
\begin{gathered}
\varepsilon_{\perp x}=\frac{a_{\perp x}-a_{\text {substrate }}}{a_{\text {substrate }}} \\
\varepsilon_{\| x}=\frac{a_{\| x}-a_{\text {substrate }}}{a_{\text {substrate }}},
\end{gathered}
$$

where $x$ represents either InAs or GaAs.

Estimates of the average perpendicular, $\epsilon_{\perp}$, and average parallel, $\boldsymbol{\epsilon}_{\|}$, strains can be determined from DCXRD rocking curves using:

$$
\begin{aligned}
\Delta \theta_{\text {measured }}= & \left(\varepsilon_{\perp \text { average }} \cos ^{2} \phi+\varepsilon_{\| \text {average }} \sin ^{2} \phi\right) \tan \theta_{B} \\
& +\left(\varepsilon_{\perp \text { average }}-\varepsilon_{\| \text {average }}\right) \sin \phi \cos \phi,
\end{aligned}
$$

where $\phi$ is the tilt and $\theta$ the Bragg angle for the substrate, and $\Delta \theta_{\text {measured }}$ is the angular separation between the substrate and zeroth order peak measured from the rocking curve. The first and second terms on the right of Eq. (7) represent the change in the Bragg angle and tilt, respectively. This equation allows the determination of $\varepsilon_{\perp \text { average }}$ and $\varepsilon_{\| \text {average }}$ from two rocking curves measured at different reflections, in our case the (004) symmetric and (115) glancing incidence, asymmetric reflections.

\section{Strain estimate from the as-grown sample}

No distinguishable strain relaxation has occurred in the as-grown sample $\left(\varepsilon_{\|}=0\right)$, so that the lattice mismatch is totally accommodated by biaxial compression of InAs. Thus substituting $d_{\mathrm{InAs}}=7.7 \AA$ and $d_{\mathrm{GaAs}}=500 \AA$ into Eq. (5) gives $\varepsilon_{\perp \text { average }}=2.2 \times 10^{-3}$. Equation (7) then gives $\Delta \theta$ $=295 \mathrm{arc} \mathrm{sec}$ which is in good agreement with the $282 \mathrm{arc}$ sec measured from the (004) rocking curve spectrum, and confirms that little macroscopic strain relaxation has occurred in the as-grown sample. However this does not rule out local strain relaxation near the QDs which is difficult to measure using DCXRD due to the small material volume involved. TEM studies are much more sensitive to any microscopic strain relaxation processes occurring in these stacked QD structures. As seen in the earlier TEM images (Fig. 5), small defect centers were present throughout the as-grown and RTA samples and may well be a strain relaxation mechanism.

\section{Strain estimate for the RTA sample}

After rapid thermal annealing the peaks in the (004) rocking curve spectrum are skewed to the right by a second set of peaks, possibly from strain relaxed material. It is difficult to distinguish this second set of peaks in the (004) reflection and a rough estimate was made for the zeroth order peak by fitting it with two Gaussians. This gave an angular separation between the relaxed zeroth order peak and the substrate peak of 273 arc sec For the (115) reflection two new tensile peaks labeled 1 and 3 are present, however the zeroth order peak associated with these two peaks is obscured by the substrate peak. The separation between peak 1 and 3 is 452 arc sec. As a rough guide we estimate the zeroth order peak to be 452 arc sec lower than peak 1 which corresponds to 99 arc sec. Using these values and Eq. (7) gives the perpendicular strain, $\varepsilon_{\perp \text { average }}=2 \times 10^{-3}$ and a parallel strain, $\varepsilon_{\| \text {average }}$, of the order of $10^{-2}$. This figure agrees qualitatively well with that calculated earlier for the two layer composite square plate and further supports the idea that the material within the threading dislocations may be responsible for the additional sets of peaks observed in the symmetric and asymmetric DCXRD spectra.

\section{CONCLUSIONS}

The influence of RTA on a 30 stacked InAs/GaAs MBE grown QDIP structure was investigated using PL, DCXRD, $\mathrm{XTEM}$, and device processing and characterization. Small defect centers close to the quantum dots are present within the as-grown and annealed samples and are believed to be a strain relaxation mechanism. After RTA no shift in the ground state QD PL emission was observed which is contrary to findings by other groups where interdiffusion of Ga and In at the QD interface led to a blueshift. However, XTEM shows that relaxation has occurred within extensive threading dislocations which are also correlated with the presence of additional peaks in the asymmetric and symmetric DCXRD scans after RTA. This supports the theory that within structures with high dislocation content, annealing tends to induce additional dislocations instead of strain induced interdiffusion. Two strong PL peaks were also ob- 
served after RTA at 1.26 and $1.04 \mathrm{eV}$ and are believed to be due to $\mathrm{Si}$ related complexes within the heavily doped GaAs bottom and top contact layers. The $1.26 \mathrm{eV}$ luminescence overlaps and obscures the PL from the QD ground state. It is therefore important not to attribute the increased PL intensity after RTA with an improved QD material quality. RTA increased the device dark current by 4 orders of magnitude. This severely degraded the device performance so that further device characteristics such as their photoresponse could not be measured. This large dark current is likely due to the formation of extensive threading dislocations and/or the $\mathrm{Si}$ related complexes in the GaAs contact layers.

\section{ACKNOWLEDGMENTS}

The research at the Australian National University was supported by the Australian Research Council and the work at the University of Michigan was supported by DARPA under Grant No. DAAD19-00-1-0394.

${ }^{1}$ V. Ryzhii, Semicond. Sci. Technol. 11, 759 (1996).

${ }^{2}$ J. Phillips, P. Bhattacharya, S. W. Kennerly, D. W. Beekman, and M. Dutta, IEEE J. Quantum Electron. 35, 936 (1999).

${ }^{3}$ L. Fu, H. H. Tan, C. Jagadish, N. Li, L. Ning, X. Liu, W. Lu, and S. C. Shen, Appl. Phys. Lett. 78, 10 (2001).

${ }^{4}$ M. B. Johnston, M. Gal, N. Li, Z. Chen, X. Liu, N. Li, W. Lu, S. C. Shen, L. Fu, H. H. Tan, and C. Jagadish, Appl. Phys. Lett. 75, 923 (1999).

${ }^{5}$ R. Leon, Y. Kim, C. Jagadish, M. Gal, J. Zou, and D. J. H. Cockayne, Appl. Phys. Lett. 69, 1888 (1996).

${ }^{6}$ S. Malik, C. Roberts, R. Murray, and M. Pate, Appl. Phys. Lett. 71, 1987 (1997).

${ }^{7}$ S. Krishna, S. Raghavan, A. L. Gray, A. Stintz, and K. J. Malloy, J. Appl. Phys. 80, 3898 (2002)
${ }^{8}$ Q. D. Zhuang, J. M. Li, X. X. Wang, Y. P. Zeng, Y. T. Wang, B. Q. Wang, L. Pan, J. Wu, M. Y. Kong, and L. Y. Lin, J. Cryst. Growth 208, 791 (2000).

${ }^{9}$ C. H. Roh, Y. J. Park, K. M. Kim, Y. M. Park, E. K. Kim, and K. B. Shim, J. Cryst. Growth 226, 1 (2001).

${ }^{10}$ L. Jiang, S. S. Lin, N.-T. Yeh, J.-L. Chyi, C. E. Ross, and K. S. Jones, Appl. Phys. Lett. 82, 1986 (2003).

${ }^{11}$ E. W. Williams and H. B. Bebb, in Semiconductors and Semimetals, Transport and Optical Phenomena, edited by R. K. Willardson and A. C. Beer (Academic, New York, 1972), Vol. 8, Chap. 5.

${ }^{12}$ J. Phillips, K. Kamath, and P. Bhattacharya, Appl. Phys. Lett. 72, 2020 (1998).

${ }^{13}$ Z. M. Wang, S. L. Feng, Z. D. Lu, Q. Zhao, X. P. Yang, Z. G. Chen, Z. Y. Zxu, and H. Z. Zheng, J. Electron. Mater. 27, 59 (1998).

${ }^{14}$ K. Stewart, M. Buda, J. Wong-Leung, L. Fu, C. Jagadish, A. Stiff-Roberts, and P. Bhattacharya, Proceedings of 2002 Conference on Optoelectronic and Microelectronic Materials and Devices (IEEE, Piscataway, NJ, 2003).

${ }^{15}$ N. H. Ky, L. Pavesi, D. Araujo, J. D. Ganiere, and F. K. Reinhart, J. Appl. Phys. 69, 7585 (1991).

${ }^{16}$ N. H. Ky and F. K. Reinhart, J. Appl. Phys. 83, 718 (1998).

${ }^{17}$ M. Suezawa, A. Kasuya, Y. Nishina, and K. Sumino, J. Appl. Phys. 69, 1618 (1990).

${ }^{18}$ M. Suezawa, A. Kasuya, Y. Nishina, and K. Sumino, J. Appl. Phys. 76, 1164 (1994).

${ }^{19}$ I. Harrison, L. Pavesi, M. Henini, and D. Johnston, J. Appl. Phys. 75, 3151 (1993).

${ }^{20}$ J. Liang, J. Jiang, J. Zhao, and Y. Gao, J. Appl. Phys. 79, 7173 (1996).

${ }^{21}$ S. K. Mehta, R. Muralidharan, G. D. Sharda, and R. K. Jain, Semicond. Sci. Technol. 7, 635 (1992).

${ }^{22}$ K. Shiramine, Y. Horisaki, D. Suzuki, S. Itoh, Y. Ebiko, S. Muto, Y. Nakata, and N. Yokoyama, Jpn. J. Appl. Phys., Part 1 37, 5493 (1998).

${ }^{23}$ K. Shiramine, Y. Horisaki, D. Suzuki, S. Itoh, Y. Ebiko, S. Muto, Y. Nakata, and N. Yokoyama, J. Cryst. Growth 205, 461 (1999).

${ }^{24}$ S. N. G. Chu, J. Electrochem. Soc. 145, 3621 (1998). 